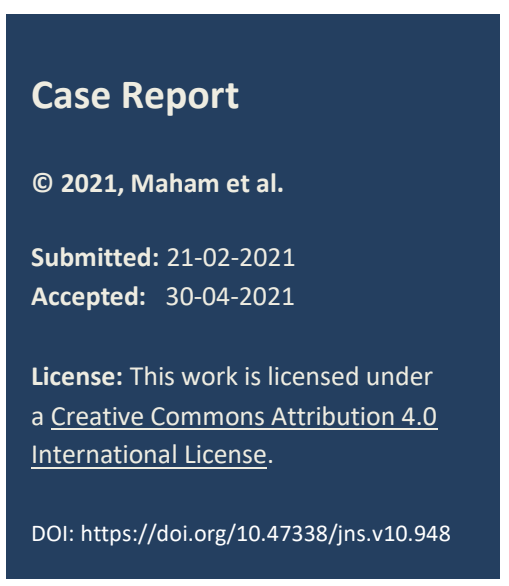

\title{
Congenital hepatic hemangioma presenting with neonatal intestinal obstruction: A case report
}

\section{Sumaira Noor Maham, ${ }^{*}$ Shabbir Ahmad, Muhammad Jawad Afzal, Nabila Talat}

Department of Pediatric Surgery Unit II, The Children's Hospital and the Institute of Child Health, Lahore

Correspondence*: Sumaira Noor Maham, Department of Pediatric Surgery Unit II, The Children's Hospital and the Institute of Child Health, Lahore. E-mail: dr.maham318@gmail.com

\section{KEYWORDS \\ Congenital hemangioma, Intestinal obstruction, Neonate \\ ABSTRACT \\ Background: Congenital hepatic hemangioma usually presents with abdominal distension. Rarely it may cause intestinal obstruction. We present a case of congenital hepatic hemangioma causing neonatal intestinal obstruction. \\ Case Presentation: A 4-day-old neonate presented with clinical and radiological features of neonatal intestinal obstruction. On exploration, a loop of jejunum was found adherent with a hepatic mass, arising from the left lobe of the liver. The hepatic mass profusely bled in an attempt of removing the adherent jejunal loop. Thus, the adherent portion was isolated and jejunojejunal end to end anastomosis was done. The hepatic mass along with an adherent small piece of jejunum was also excised. Histopathology showed hepatic hemangioma. The patient is doing fine on the 10-month follow-up. \\ Conclusion: We report a rare presentation of congenital hepatic hemangioma with neonatal intestinal obstruction. Although the optimum therapy for hepatic hemangioma is medical management, at times, surgical resection becomes a necessary option.}

\section{INTRODUCTION}

Congenital hemangioma is "Hemangioma with inutero proliferation period, fully formed at birth "(ISSVA classification).[1] It is sub-classified as rapidly involuting, non-involuting, and partially involuting.[2] Hepatic hemangioma (congenital and infantile) is a benign vascular tumor. The clinical behavior can be innocuous or life-threatening.[1] Most commonly present before the age of 6 months as an abdominal mass with or without congestive cardiac failure or consumptive thrombocytopenia. Rarely, the hepatic hemangioma may present with vomiting and gastric outlet obstruction.[3] Hemangioma presenting as a gastric outlet and duodenal obstruction has also been reported in the literature.[4,5] We decided to report this case because congenital hepatic hemangioma seldom presents with intestinal obstruction, and at times the resection of hepatic hemangioma becomes necessary in neonatal life.

\section{CASE REPORT}

A 4-day-old term female neonate born through the cesarean section to a primigravida mother with unremarkable antenatal history, weighing $3.2 \mathrm{~kg}$, present- ed with complaints of vomiting and abdominal distension soon after the birth. The vomiting was aggravated after starting the feed, greenish-yellow in color, and in multiple episodes. On examination, the baby was afebrile with a respiratory rate of $42 / \mathrm{min}$, and heart rate of $138 / \mathrm{min}$. The abdomen was distended with palpable bowel loops in the paraumbilical region, bowel sounds were absent.

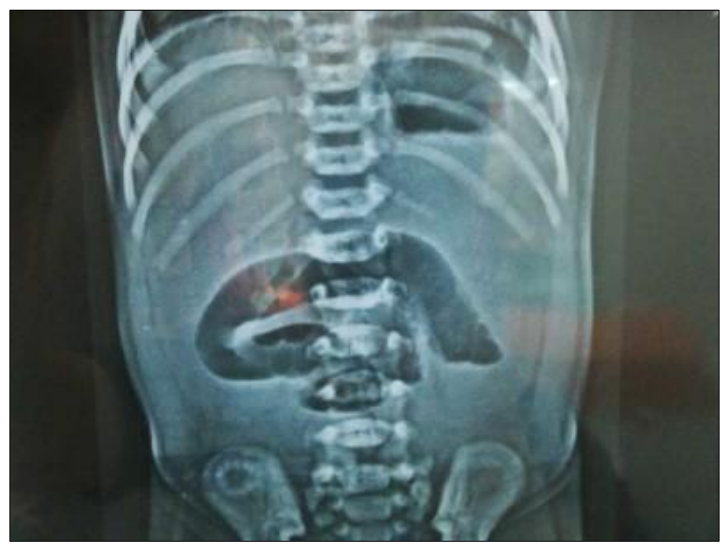

Figure 1: X-ray showing intestinal obstruction

The baby was admitted, and a nasogastric tube was passed which yielded greenish aspirate. X-ray abdo- 
men erect showed a few dilated loops of small bowel in the mid-abdomen; gas in the colon and rectum was not visualized (Fig.1). Ultrasound of abdomen documented gaseous distension of the small bowel. All baseline investigations were done which turned out as normal.

The baby underwent exploratory laparotomy with a diagnosis of congenital neonatal intestinal obstruction most likely small bowel atresia. On exploration, the small bowel was found distended till the mid jejunum, at that point the next few inches of the jejunum were densely adherent with a congested pedunculated hepatic mass $(6 \times 8 \mathrm{~cm})$ arising from the left lobe of the liver. The adherent jejunum was found twisted and ischemic, causing a complete intestinal obstruction (Fig.2). We suspected the mass was hepatic hemangioma. It started to bleed profusely in an attempt to separate it from the adherent jejunum, therefore, the jejunum was resected just proximal to its attachment with the hepatic mass on either side and an end-toend jejunal anastomosis was performed. Since the necrotic jejunum could not be freed from the mass, therefore we decided to excise the hepatic mass. The excision was started with a harmonic scalpel, but that harmonic shear started malfunction mid-way the resection. The rest of the resection was performed with the help of bipolar cautery. As the mass was pedunculated, therefore, no operative difficulty was encountered. The cut edge of the left lobe of the liver was secured with stitches over Surgicel ${ }^{\circledR}$ as a pledget.

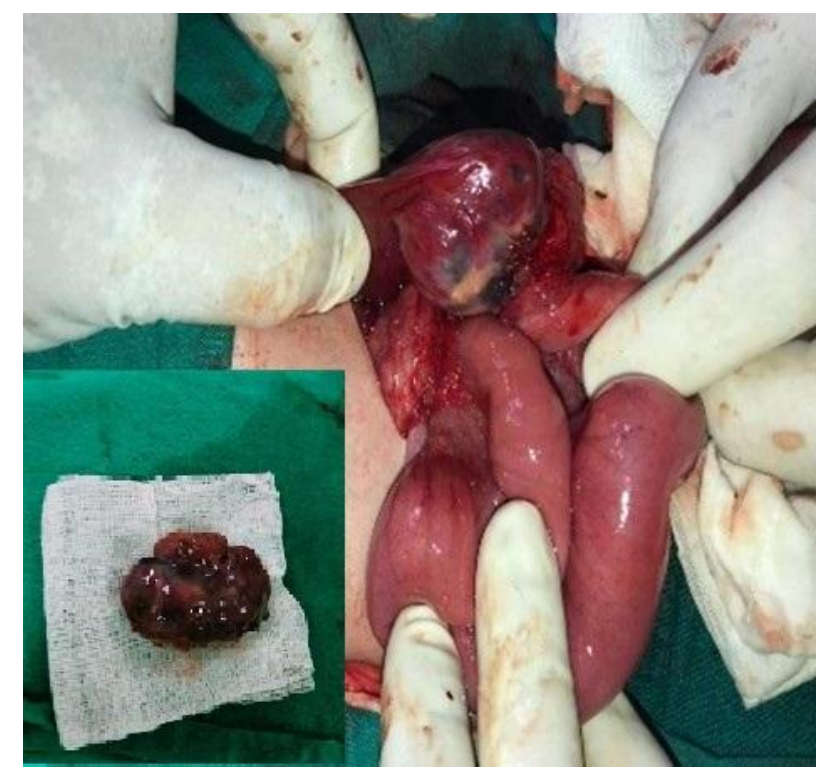

Figure 2: Liver mass with adherent small bowel. Inset shows resected lesion.

The initial postoperative course remained uneventful however the baby developed wound infection and became septic. Antibiotics were upgraded along with wound management. The cultures of the wound and blood did not yield any organism. The patient responded well and discharged on the 12 th postopera- tive day. Biopsy report followed suggestive of liver hemangioma. At a 10-month follow-up, the baby is thriving well and achieving all developmental milestones normally. Follow-up ultrasound showed no recurrence of the hepatic mass

\section{DISCUSSION}

Progress has been made in the field of vascular anomalies over the period of decades, leading to more refined classifications, genetic etiologies, associated complications, congenital malformations, and diagnostic and therapeutic techniques. The occurrence of hemangioma has commonplace in pediatric practice, liver hemangiomas are the most frequently encountered as primary benign tumors in the liver, but congenital liver hemangiomas have rarely been reported with intestinal obstruction in neonates and adults. Zhou JX et al. also reported a case of 21 years old male having hepatic hemangioma with intestinal obstruction.[7] Because most liver hemangiomas remain asymptomatic, they are often recognized incidentally on radiologic tests for other reasons. As in our case, had there not been an intestinal obstruction, it would not have been presented in neonatal life. In infants, hepatic hemangiomas are benign tumors that usually appear during the 1st 6 months of life, however high mortality is associated with Kasabach - Merritt syndrome. A hepatic hemangioma can be focal, multifocal, and pedunculated; in our case it was pedunculated.

Its presentation with recurrent vomiting is rarely mentioned [3]; only one case with abdominal lump and bilious vomiting has been reported by Sinha et al. Their differential diagnoses were duodenal stenosis and midgut volvulus; on exploration, liver hemangioma found causing obstruction of the third part of the duodenum.[3] Abdulrahman et al. reported its presentation as a large intra-abdominal cyst on antenatal scan and in the immediate postnatal period development of respiratory distress and abdominal compartment syndrome.[6]

Prenatal diagnosis can be suspected on fetal ultrasound scan showing abdominal mass or hydrops and postnatal with ultrasounds, CT scan; MRI is more specific for extent and nature of the lesion, obviating the need for angiography. In the index case, no prenatal diagnosis was available on the antenatal scan. Due to the risk of hemorrhage biopsy is not recommended. Although angiography is the gold standard in the diagnosis of hemangioma; the greatest disadvantage is its invasiveness. Serum alpha-fetoprotein levels were traditionally used as tumor markers for hepatoblastoma and to differentiate it from hepatic hemangioma, elevated alpha-fetoprotein levels have never been reported with hepatic hemangioma.[3] But in our case, the clinical presentation was acute, and the radiographs suggested small bowel obstruction, 
therefore, such investigations could not be performed. Though ultrasound was performed, it could not identify hepatic mass as it is largely operator dependent modality.

ISSVA [1] recommends Doppler ultrasound for liver hemangioma as 1 st imaging modality at presentation, larger hemangiomas need differentiation from hepatoblastoma and metastatic pediatric tumors (neuroblastoma); Doppler ultrasound adds valuable information in larger lesions. But in our case, the patient has an acute presentation with intestinal obstruction so no Doppler was done. On targeted MRI, both congenital and infantile hepatic hemangioma is hyperintense on T2 weighted images. On T1 weighted images are hypointense relative to normal liver parenchyma. Intralesional calcifications are exclusively described in congenital hemangiomas.[1] In our case, the biopsy report also showed numerous vascular channels with necrosis and calcification; suggestive of congenital hepatic hemangioma.

According to the natural history of hepatic hemangioma (both congenital and infantile hepatic hemangioma), spontaneously involute and no treatment is required in incidental cases. Treatment is required for symptomatic lesions. Commonly used treatment options are medical management with propranolol, steroids, and alpha-interferon as they inhibit the proliferation of smooth muscles and endothelial cells. Hepatic resection has been traditionally recommended for the management of ruptured hemangioma and con-

\section{REFERENCES}

1. Iacobas I, Phung TL, Adams DM, Trenor III CC, Blei F, Fishman DS, et al. Guidance document for hepatic hemangioma (infantile and congenital) evaluation and monitoring. J Pediatr. 2018; 203:294-300.

2. ISSVA classification of vascular anomalies (c) 2018. International Society for the Study of Vascular Anomalies. Available at https://issva.org/classification. Accessed [27.4.2021]

3. Sinha A, Ramji S, Sarin YK. Neonatal hepatic hemangioendothelioma: Unusual presentations. Indian Pediatr. 2001; 38:1052-5.

4. Karatzas T, Smirnis A, Dimitroulis D, Patsouras D, Evaggelou K, Kyklos S, et al. Giant pedunculated siderably malignant lesions. We resected the tumor because it was adherent to the gut, causing obstruction and gangrene of the gut, and bled profusely on dissection.

Hepatic artery embolization can also be done in symptomatic bilobar hepatic hemangiomas. Chemotherapy and radiotherapy for liver hemangioma have previously shown no good results.

Resection of liver hemangioma has been previously done for solitary symptomatic hemangiomas and proven to have a good outcome.[6] Our study case has also proven that complete resection of symptomatic localized pedunculated hemangioma along with resection of gangrenous gut and end to end anastomosis at the same time results in relief of acute symptoms along with good prognosis and no residual disease in certain situations when there is no way out other than resection.

\section{Acknowledgements: Nil}

Conflict of Interest: NT is part of editorial team, however, the manuscript was independently handled by another editor and she was not involved in decision making about this manuscript.

\section{Source of Support: Nil}

Consent to Publication: Author(s) declared taking informed written consent for the publication of clinical photographs/material (if any used), from the legal guardian of the patient with an understanding that every effort will be made to conceal the identity of the patient, however it cannot be guaranteed.

Author Contributions: Author(s) declared to fulfil authorship criteria as devised by ICMJE and approved the final version. hepatocellular carcinoma with hemangioma mimicking intestinal obstruction. BMC Gastroenterol. 2011; 11:15 .

5. Aydin C, Akbulut S, Kutluturk K, Kahraman A, Kayaalp $\mathrm{C}$, Yilmaz S. Giant hepatic hemangioma presenting as gastric outlet obstruction. Int Surg. 2013; 98:19-23.

6. Almuawi A, Daboos M, Prasad R. Giant pedunculated hepatic hemangioma necessitating surgical excision in a neonate: A case report. J Neonatal Surg. 2020; 9:15.

7. Zhou JX, Huang JW, Wu H, Zeng Y. Successful liver resection in a giant hemangioma with intestinal obstruction after embolization. World J Gastroenterol: WJG. 2013; 19:2974. 